\title{
Anti-rheumatic drugs
}

\author{
H. L. F. CURREY \\ M.Med., F.R.C.P. \\ The London Hospital Medical College, London
}

\begin{abstract}
Summary
Prescription of anti-rheumatic drugs should be by approved name because in most cases differences in absorption can be compensated by titration of dosage against effectiveness and toxicity. There are a few instances, for example phenylbutazone, where known differences of bio-availability justify the use of a particular brand.
\end{abstract}

THE vast quantity of drugs prescribed for rheumatic disorders, many of them both expensive and potentially very harmful, demand efficient and safe prescribing habits. The list of drugs shown in Table 1 is a personal one and is an indication only of the range of drugs prescribed for treating specifically rheumatological problems. Amongst these drugs there are only a few examples where factors such as bio-availability or 'patient acceptability' are thought to differ significantly between the different proprietary preparations available, so that any departure from a general policy of prescribing by approved name needs to be defended. There are a few general, and some specific, considerations which provide at least a talking point in this regard.

TABLE 1. Some drugs used in rheumatology

\begin{tabular}{ll}
\hline $\begin{array}{l}\text { Aspirin } \\
\text { Soluble aspirin } \\
\text { Enteric coated asprin }\end{array}$ & Prednisolone pivalate inj. \\
Aloxiprin & Corticotrophin gelatin inj. \\
& Sodium aurothiomalate inj. \\
Paracetamol & Chloroquine sulphate \\
Ibuprofen & Hydroxychloroquine sulphate \\
Benorylate & D-Penicillamine \\
Dextropropoxyphene & Cyclophosphamide \\
Codeine & Chlorambucil \\
Dihydrocodeine & Azathioprine \\
Phenylbutazone & Colchicine \\
Oxyphenbutazone & Allopurinol \\
Indomethacin & Probenecid \\
Prednisone & Ethibenecid \\
Prednisolone & Sulphinpyrazone \\
Enteric coated prednisolone & \\
Prednisolone phosphate & \\
\hline
\end{tabular}

It has been argued that chronic rheumatic disorders in which drug therapy has been stabilized can conveniently be treated with mixtures of drugs prepared in one formulation. Such mixtures would indeed be more conveniently referred to by short trade names; however, the case for the use of such combined preparations in rheumatology is certainly not established. One could possibly make a case for preparations containing (for example) both allopurinol and colchicine, but it is doubtful whether such fixed-proportion preparations are really desirable. A number of proprietary mixtures are promoted for rheumatic diseases, of which some (e.g. mixtures of salicylates and steroids or of phenylbutazone and amidopyrine) represent frank irresponsibility on the part of the manufacturers. Admittedly the logistics of long-term out-patient drug therapy for rheumatological patients raise difficult issues, but it seems unlikely that drug mixtures will do anything other than compound the problems. Conceivably, the answer may be combined presentation of the individual tablets in something like 'blister packs' which will display the appropriate array of pills to be taken at particular times each day. Such a system would need to be versatile, with the drug programme tailored to individual requirements. And if the prescribing doctor is going to keep control of such therapy it is probably a situation in which one should insist on adhering to approved names for at least most of the individual drugs.

Amongst the solid, oral dosage forms of the drugs listed above, it has been suggested that the formulation of the following affects their biological availability (Davis, 1972). Aspirin (Levy, 1961), indomethacin, paracetamol (Gwilt et al., 1963), phenylbutazone (Searl and Pernarowski, 1967), prednisolone and prednisone.

At present in the United Kingdom both enteric coated aspirin and aloxiprin tablets are each available only as one proprietary brand, so that the prescriber knows exactly what will be dispensed when he uses these approved names. This is not so in the case of ordinary aspirin or soluble aspirin tablets, different proprietary brands of which differ in such 
matters as particle size and, hence, particularly, in rate of absorption (Levy, 1961). For complete consistency of response, therefore, a brand name has to be specified. However, in practice, the method of employing salicylates, in which the dosage is titrated roughly against clinical response and symptoms of over-dosage, the ready availability of blood salicylate determinations and the degree of variation between different products probably do not constitute sufficient grounds for insisting on particular proprietary brands. More information is needed about the influence of the rate of disintegration of aspirin tablets on gastric bleeding. A separate issue is the fact that some patients have strong preferences between different brands of soluble aspirin, based on taste and consistency. This may justify specifying a brand name. Indomethacin is at present marketed in the United Kingdom only as one brand, so this problem does not arise yet.

Paracetamol is marketed in this country as a number of different proprietary brands and there is certainly considerable variation in absorption patterns, both between different products and between different subjects taking the same product (Gwilt et al., 1963). This has stimulated Winthrop Laboratories to produce paracetamol in a tablet ('Panasorb') with a special sorbitol-containing base. This, it is claimed, goes a long way towards eliminating the inter-patient variation. It is for the individual prescriber to decide whether the more consistent blood levels claimed for this product justify prescription by proprietary name. Such a policy would not increase the cost of treatment unduly. On the other hand this is not a type of therapy in which either dosage or blood levels are known to be particularly critical.

Phenylbutazone is also available as a variety of proprietary brands and, again, these have been shown to vary considerably both in drug content and in biopharmaceutical properties (Searl and Pernarowski, 1967). As this is a powerful drug with potentially very serious short-term and long-term toxicity, it is mandatory for the prescriber to be aware of these variations. At the present time there would seem to be an argument in favour of specifying 'Butazolidin' brand of phenylbutazone. This is the product of Geigy, who originally developed the drug. It is also the preparation used in the original clinical trials on which this form of therapy is based, and the technical quality of the tablets is generally thought to be satisfactory. Against this must be set the fact that it is considerably more expensive than some other preparations on the market.
Adrenal corticosteroids constitute another group of drugs in which differences in tablet formulation influence absorption. However, in the case of the tablets most commonly used in rheumatologyprednisolone-it seems unlikely that, amongst products now on the market, these differences are important, and price differences are not great.

Long-acting preparations of corticotrophin for injection, for example corticotrophin in hydrolysed gelatin, probably vary somewhat both in the physical properties which determine ease of injection and also in rate of absorption, and, hence, pharmacological response. Here it is consistency of response that is critical and, having found a brand which is acceptable for self-administration, it is probably advisable to continue specifying the same proprietary preparation.

Amongst the drugs listed in Table 1 some of the approved names are at a disadvantage in having to compete with more convenient trade names. Patients, at least, find it easier to ask for 'Panadol' rather than paracetamol and 'Imuran' rather than azathioprine. In the case of the antimetabolite this probably constitutes a reason for providing such patients with a drug card on which both names are specifiedwhatever is written on the prescription. The pros prietary name 'DF 118 ' is widely used by both $\vec{\varphi}$ patients and doctors as a convenient description for $f$ dihydrocodeine. Such numerical identification migkf conceivably introduce confusion with tablet strength, dosage or quantity to be supplied, and is probably better avoided in prescription writing.

In conclusion, prescription of anti-rheumatic drugs should probably always be by approved name but, at the discretion of the prescribing doctor, there are perhaps a few examples in which a particular brand should be stipulated.

\section{Acknowledgment}

I am indebted to members of the pharmaceutical staff, The London Hospital, for advice in preparing this paper.

\section{References}

Davis, S.S. (1972) Scientific principles in design of drug dosage formulations. British Medical Journal, 1, 102.

Gwilt, J.R., Robertson, A., Goldman, L. \& BlanChard, A.W. (1963) The absorption characteristics of paracetamol tablets in man. Journal of Pharmacy and Pharmacology, 15, 445.

LEVY, G. (1961) Comparison of dissolution and absorption rates of different commercial aspirin tablets. Journal of Pharmaceutical Sciences, 50, 389.

Searl, R.O. \& Pernarowski, M. (1967) The biopharmaceutical properties of solid dosage of forms: 1 . An evaluation of 23 brands of phenylbutazone tablets. Canadian Medical Association Journal, 96, 1513. 


\section{Discussion}

Dr B. W. Cromie emphasized that he would only attempt to justify combinations of drugs which the patient needs; the use of unnecessary drugs in combinations could not be defended.

Dr H. L. F. Currey cited the example of analgesic nephropathy, suggesting that confusion about the aetiology of this condition had resulted from the widespread use of combination analgesics, so that the individual compound responsible could not be readily identified. 